\title{
EVALUACIÓN DE UN MÉTODO PARA LA ENSEÑANZA DE LENGUAS EXTRANJERAS
}

\author{
Lilliam Cascante Madrigal
}

\begin{abstract}
Resumen: El presente articulo tiene por objetivo analizar la experimentación que se llewi a cabo con grupos de alumnos de secundaria, para evaluar el método de francés La Fenêtre, elaborado por Ligia Salas Pereira. Es la primera vez que un método para la enseñanza del francés lengua extranjera se experimenta y evalua antes de ser implantado en el nivel nacional. Durante la experimentación se aplicaron cliferentes instrumentos, tanto a los alumnos de las instituciones inuolucradas como a sus profesores. Los resultados finales fueron muy satisfactorios pues reflejaron un nivel de adquisición de conocimientos semejante al que se obtiene con la utilización de métodos elaborados por especialistas franceses.
\end{abstract}

\section{Introducción}

Desde hace varios años se han utilizado métodos o manuales importados de otros países, para enseñar idiomas extranjeros en las instituciones de Enseñanza Secundaria de nuestro país. De acuerdo con Galisson y Coste (1976), se entiende por método "la suma de pasos razonados, basados en un conjunto coherente de principios o de hipótesis lingüísticas, psicológicas, pedagógicas y que respondan a un objetivo determinado."

Un método extranjero no garantiza, por lo tanto, la aplicación de objetivos, contenidos y estrategias acordes con la realidad educativa del país, razón por la cual es importante pensar en una experimentación y una evaluación de ellos, antes de su implementación en las aulas, en el nivel nacional. Con esta acción se podría observar la eficiencia de dichos materiales didácticos, antes de recomendarlos para el uso general.

En Costa Rica se han utilizado una variedad de manuales para la enseñanza del francés:

a. Los llamados métodos tradicionales, con los que los alumnos aprenden estructuras gramaticales o léxico aislado de un contexto, tales como: "C'est la table" (Es la mesa).

b. Los métodos directos, con los que se trata de dar un medio de comunicación, pero en situaciones artificiales, por ejemplo: diálogos pre-elaborados, que el estudiante memoriza, pero que no lo preparan para resolver una situación de comunicación real y espontánea. 
c. Los métodos audio-visuales, los cuales no resultaron muy exitosos en los liceos de Costa Rica por su alto costo y por las limitaciones materiales de las instituciones, a pesar de sus ventajas ya que presentan personajes y diálogos en situaciones de comunicación.

d. Los métodos comunicativos que se han utilizado en los últimos años, basados en situaciones de comunicación auténticas. En estos métodos se introducen factores cognoscitivos, psicológicos y socioculturales, además del factor lingüístico.

Conociendo la problemática que se le presenta a menudo a la Asesoría Nacional de Francés del Ministerio de Educación Pública, para seleccionar el método más adecuado para enseñar la lengua en cuestión, se aprovechó el hecho de que el método de francés La Fenêtre, de Ligia Salas Pereira, había sido elaborado en Costa Rica y para Costa Rica, para realizar, en la Universidad de Costa Rica, con el apoyo del Instituto de Investigación para el Mejoramiento de la Educación Costarricense (IIMEC) y la Escuela de Formación Docente, un proyecto para su experimentación y su evaluación. Este método, se presenta "como un aporte lingüístico, cultural y formativo para la enseñanza y aprendizaje del francés en Costa Rica, ya que toma en cuenta la realidad institucional de nuestro país" (Cascante y Salas 1991). El proyecto se realiza para determinar si el método:

"logra una mejor actitud hacia el francés, comparado con los otros manuales utilizados,

logra un mejor rendimiento en francés, permite el mejor aprovechamiento del tiempo y del espacio en el aula y mayor interacción entre alumno y profesor." (Cascante y Salas, p.4, 1994).

\section{Etapas de la evaluación del método}

Con el fin de evaluar el método se propusieron las siguientes etapas:

1. Medir la actitud de los estudiantes de sétimo año de secundaria hacia el aprendi- zaje del francés, utilizando las escalas bipolares de diferencial semántico. La actitud, según Anastasi, citado por Hernández y Matarrita (1989), se define como "una tendencia a reaccionar favorable o desfavorablemente hacia una clase determinada de estímulos como un grupo social, una costumbre o una institución."

2. Evaluar la utilización del tiempo de lección, del espacio del aula y de la participación de profesor y alumnos, con base en la información recopilada utilizando una guía de observación de lecciones, en la cual se toman las estrategias de enseñanza, en cuenta el tiempo de exposición del profesor, la utilización de materiales didácticos, la interacción en el aula, el ambiente, la motivación de los alumnos y el espacio físico en cada una de las instituciones.

3. Evaluar el rendimiento de los estudiantes, es decir, la adquisición de conocimientos sobre el uso de la lengua francesa, mediante la aplicación de una prueba escrita, con enfoque comunicativo, con 50 ítemes de selección para lograr más objetividad en los resultados.

\section{Elaboración de los instrumentos}

Para desarrollar el trabajo descrito en las diferentes etapas se elaboraron los siguientes instrumentos:

A. - un pre-test y un post-test, con la utilización de escalas bipolares de diferencial semántico, para medir la actitud de los alumnos hacia el francés. Según Díaz y Salas (1970), "El diferencial semántico (D.S.) no es un test psicológico en el sentido de reactivos, calificativos y baremos específicos. Constituye, sí, una técnica de medida muy generalizable que exige adaptación de su forma y contenido según el tipo de problema que se quiere investigar".

En este caso, el instrumento contiene nueve escalas bipolares para medir tres dimensiones, cada una con tres escalas: EVALUACION con las escalas de Agradable-Desagradable, Feo-Bonito y Fácil- 
Difícil; POTENCIA con las escalas de Aburrido-Entretenido, Complicado-Sencillo, Descansado-Cansado y la dimensión ACTIVIDAD con Util-Inútil, Activo-Pasivo y Necesario-Innecesario.

B. - una guía para realizar la observación de lecciones, con el fin de obtener datos sobre todo el desarrollo de la lección, tomando en cuenta elementos relacionados con el aprovechamiento del tiempo y del espacio en el aula, como por ejemplo: desplazamiento del profesor y empleo de los materiales didácticos, así como sus estrategias de enseñanza; trabajo del alumno en la clase y sus estrategias de aprendizaje; interacción profesor-alumno, alumno-profesor, alumno-alumno.

C. - una prueba con referencia a criterios para determinar el dominio de los conocimientos estudiados. Según Wardrop, et al. 1982, una prueba elaborada con este modelo "sirve para diagnosticar los conocimientos específicos que un individuo posee, o la eficiencia de un programa educativo específico".

\section{Aplicación de los instrumentos}

La muestra con la cual se trabajó consta de 703 alumnos de sétimo año de la Educación General Básica y de once profesores, graduados en alguna de las universidades estatales. repartidos en once instituciones de diferentes regiones del país. Se contó con la participación voluntaria de estos profesores y sus alumnos por lo que no son todas las regiones del país las que están representadas en la muestra. Para realizar la experimentación, cada profesor fue responsable de dos grupos de alumnos de su institución, escogiddos al azar, uno "control" (utilizando otros métodos) y ouro "experimental" (utilizando el método La Fenêtre).

Durante un año lectivo, se realizaron tres o cuatro visitas a cada institución para observar lecciones, aplicar los tests o aplicar la prueba escrita de conocimientos del francés. En cada visita el observador utilizaba la guía de observación de lecciones descrita anteriormente.

\section{Resultados de la aplicación de los instrumentos}

\section{A. Del diferencial semántico}

Como se mencionó anteriormente, mediante un pre-test y un post-test de diferencial semántico, se midió la actitud hacia el francés por parte de la muestra de alumnos de los once colegios. En los resultados generales de dichos tests (Gráficos 1 y 2) vemos que en el post-test hay un descenso en todas las escalas que se midieron, pero se muestra, claramente, que en todos los grupos la actitud hacia el francés fue positiva, con mayor énfasis en los grupos experimentales. En general, los alumnos consideran la lengua francesa como útil, necesaria y agradable. En otros casos, se ven cercanos al cero conceptos como: cansado, difícil y complicado, en cuyo caso, de acuerdo con el tipo de conceptos medidos, podría decirse que existe una estrecha relación entre la metodología utilizada por los profesores para la enseñanza de una lengua extranjera y la percepción de los alumnos. Este aspecto deberá investigarse más a fondo, por parte del Ministerio de Educación Pública como parte de la evaluación de un docente.

Los grupos de alumnos fueron escogidos al azar, por lo cual sus características son diferentes, unos considerados en sus instituciones como grupos "problema" o "difíciles" por su rendimiento o por su disciplina, y otros calificados como los mejores por las mismas razones.

Gráfico 1

Resultados del diferencial semántico (Pretest)

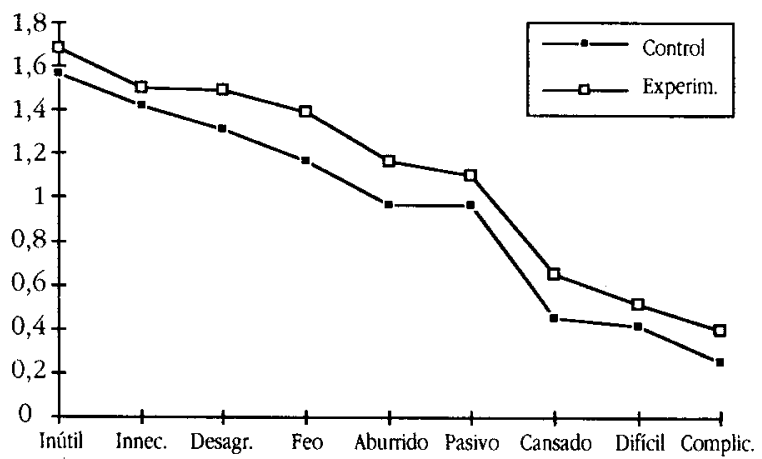


Gráfico 2

Resultados del diferencial semántico (Postest)

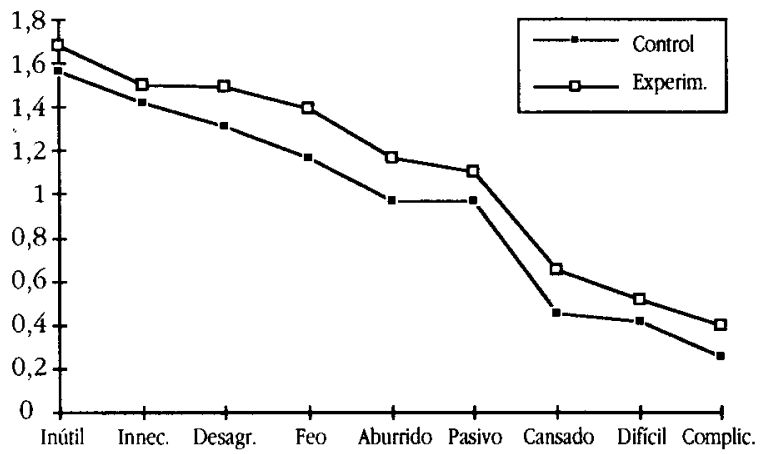

Es importante también observar que, tanto en el pre-test como en el pos-test, la diferencia entre la escala agradable y dificil, aparentemente es contradictoria, pues los alumnos perciben el francés como agradable pero no como muy fácil. En el momento de aplicar los instrumentos los estudiantes tenían solamente seis horas de clases de francés y era una lengua extranjera totalmente nueva para ellos.

\section{B. De la observación de clases}

Durante las visitas a los colegios que participaron en la experimentación, las dos investigadoras del proyecto realizaron observaciones de clase, tanto en los grupos control como en los experimentales y con el instrumento respectivo recopilaron la información.

Algunos resultados de estas observaciones que podrían estar relacionados con la formación metodológica de los profesores, son los siguientes: de los profesores observados, un $70 \%$ desarrolló sus lecciones utilizando sólo libro y pizarra, ninguno utilizó metalenguaje; un $84 \%$ respondió a los alumnos solamente en español, también un $84 \%$ mantuvo los pupitres en filas, un $58 \%$ hizo trabajar a sus alumnos en forma escrita e individualmente, y solo un 11\% organizó a sus alumnos en sub-grupos.

\section{De la prueba de rendimiento}

La prueba de rendimiento académico en francés se elaboró con referencia a cri- terios, como se explicó en el apartado de la elaboración de los instrumentos, y estuvo basada en situaciones de comunicación auténticas en francés. De acuerdo con el análisis de la prueba, se puede decir que se obtiene el mismo nivel de adquisición lingüística con los métodos franceses que fueron utilizados por los profesores en los grupos control y con el método La Fenêtre en experimentación. En ambos casos, un $60 \%$ de los alumnos obtuvo el promedio de 60 , o superior a 60 , que era la nota mínima en ese momento. Durante todo un curso lectivo se trabajó muy de cerca con este grupo de docentes que formó parte de la investigación, desarrollando diferentes aspectos, como por ejemplo capacitación sobre metodología comunicativa. Se pudo así constatar el escaso conocimiento y la poca o ninguna experiencia anterior para aplicar dicha métodología, lo que influyó para no obtener mejores resultados con La Fenêtre, cuyos fundamentos son comunicativos.

\section{Conclusiones}

Con este estudio se logró demostrar que el método La Fenêtre es otra opción con la que se logra una actitud positiva hacia el francés y un buen aprovechamiento.

Se pudo detectar, gracias a este proyecto, otras situaciones que interfieren en el desarrollo normal del programa del curso, entre ellas: reuniones, Consejos de Profesores u otras actividades no académicas en tiempo lectivo, así como situaciones que dificultan las posibilidades de mejoramiento académico de los docentes de secundaria, entre las que se pueden mencionar:

Falta de permisos para los profesores, para asistir a reuniones académicas o seminarios con especialistas en la materia, por considerarse como pérdida de "tiempo".

Falta de presupuesto para proyectos relacionados con el mejoramiento de la educación.

Poco conocimiento sobre la importancia del aprendizaje de lenguas extranjeras. 
Difícil; POTENCIA con las escalas de Aburrido-Entretenido, Complicado-Sencillo, Descansado-Cansado y la dimensión ACTIVIDAD con Util-Inútil, Activo-Pasivo y Necesario-Innecesario.

B. - una guía para realizar la observación de lecciones, con el fin de obtener datos sobre todo el desarrollo de la lección, tomando en cuenta elementos relacionados con el aprovechamiento del tiempo y del espacio en el aula, como por ejemplo: desplazamiento del profesor y empleo de los materiales didácticos, así como sus estrategias de enseñanza; trabajo del alumno en la clase y sus estrategias de aprendizaje; interacción profesor-alumno, alumno-profesor, alumno-alumno.

C. - una prueba con referencia a criterios para determinar el dominio de los conocimientos estudiados. Según Wardrop, et al. 1982, una prueba elaborada con este modelo "sirve para diagnosticar los conocimientos específicos que un individuo posee, o la eficiencia de un programa educativo específico".

\section{Aplicación de los instrumentos}

La muestra con la cual se trabajó consta de 703 alumnos de sétimo año de la Educación General Básica y de once profesores, graduados en alguna de las universidades estatales, repartidos en once instituciones de diferentes regiones del país. Se contó con la participación voluntaria de estos profesores y sus alumnos por lo que no son todas las regiones del país las que están representadas en la muestra. Para realizar la experimentación, cada profesor fue responsable de dos grupos de alumnos de su institución, escogiddos al azar, uno "control" (utilizando otros métodos) y otro "experimental" (utilizando el método La Fenêtre).

Durante un año lectivo, se realizaron tres o cuatro visitas a cada institución para observar lecciones, aplicar los tests o aplicar la prueba escrita de conocimientos del francés. En cada visita el observador utilizaba la guía de observación de lecciones descrita anteriormente.

\section{Resultados de la aplicación de los instrumentos}

\section{A. Del diferencial semántico}

Como se mencionó anteriormente, mediante un pre-test y un post-test de diferencial semántico, se midió la actitud hacia el francés por parte de la muestra de alumnos de los once colegios. En los resultados generales de dichos tests (Gráficos 1 y 2) vemos que en el post-test hay un descenso en todas las escalas que se midieron, pero se muestra, claramente, que en todos los grupos la actitud hacia el francés fue positiva, con mayor énfasis en los grupos experimentales. En general, los alumnos consideran la lengua francesa como útil, necesaria y agradable. En otros casos, se ven cercanos al cero conceptos como: cansado, difícil y complicado, en cuyo caso, de acuerdo con el tipo de conceptos medidos, podría decirse que existe una estrecha relación entre la metodología utilizada por los profesores para la enseñanza de una lengua extranjera y la percepción de los alumnos. Este aspecto deberá investigarse más a fondo, por parte del Ministerio de Educación Pública como parte de la evaluación de un docente.

Los grupos de alumnos fueron escogidos al azar, por lo cual sus características son diferentes, unos considerados en sus instituciones como grupos "problema" o "difíciles" por su rendimiento o por su disciplina, $y$ otros calificados como los mejores por las mismas razones.

Gráfico 1

Resultados del diferencial semántico (Pretest)

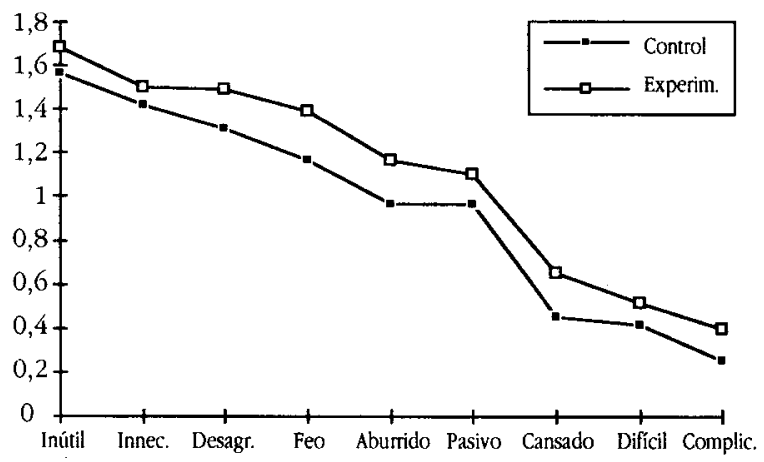


Gráfico 2

Resultados del diferencial semántico (Postest)

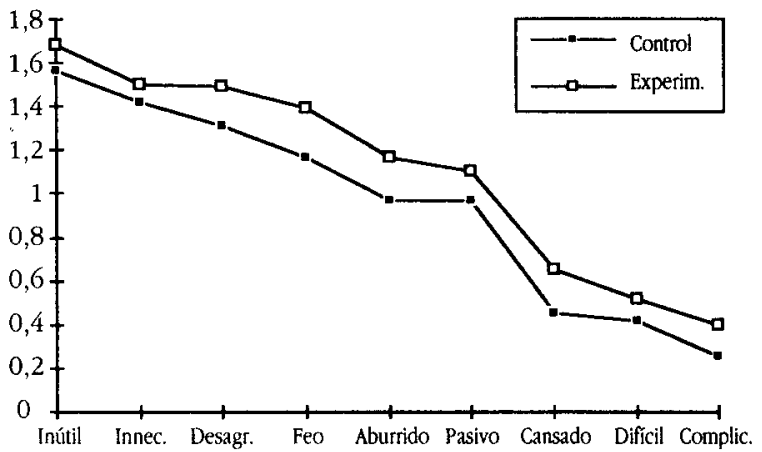

Es importante también observar que, tanto en el pre-test como en el pos-test, la diferencia entre la escala agradable y difícil, aparentemente es contradictoria, pues los alumnos perciben el francés como agradable pero no como muy fácil. En el momento de aplicar los instrumentos los estudiantes tenían solamente seis horas de clases de francés y era una lengua extranjera totalmente nueva para ellos.

\section{B. De la observación de clases}

Durante las visitas a los colegios que participaron en la experimentación, las dos investigadoras del proyecto realizaron observaciones de clase, tanto en los grupos control como en los experimentales y con el instrumento respectivo recopilaron la información.

Algunos resultados de estas observaciones que podrían estar relacionados con la formación metodológica de los profesores, son los siguientes: de los profesores observados, un $70 \%$ desarrolló sus lecciones utilizando sólo libro y pizarra, ninguno utilizó metalenguaje; un $84 \%$ respondió a los alumnos solamente en español, también un $84 \%$ mantuvo los pupitres en filas, un $58 \%$ hizo trabajar a sus alumnos en forma escrita e individualmente, y solo un 11\% organizó a sus alumnos en sub-grupos.

\section{De la prueba de rendimiento}

La prueba de rendimiento académico en francés se elaboró con referencia a cri- terios, como se explicó en el apartado de la elaboración de los instrumentos, y estuvo basada en situaciones de comunicación auténticas en francés. De acuerdo con el análisis de la prueba, se puede decir que se obtiene el mismo nivel de adquisición lingüística con los métodos franceses que fueron utilizados por los profesores en los grupos control y con el método La Fenêtre en experimentación. En ambos casos, un $60 \%$ de los alumnos obtuvo el promedio de 60 , o superior a 60 , que era la nota mínima en ese momento. Durante todo un curso lectivo se trabajó muy de cerca con este grupo de docentes que formó parte de la investigación, desarrollando diferentes aspectos, como por ejemplo capacitación sobre metodología comunicativa. Se pudo así constatar el escaso conocimiento y la poca o ninguna experiencia anterior para aplicar dicha métodología, lo que influyó para no obtener mejores resultados con La Fenêtre, cuyos fundamentos son comunicativos.

\section{Conclusiones}

Con este estudio se logró demostrar que el método La Fenêtre es otra opción con la que se logra una actitud positiva hacia el francés y un buen aprovechamiento.

Se pudo detectar, gracias a este proyecto, otras situaciones que interfieren en el desarrollo normal del programa del curso, entre ellas: reuniones, Consejos de Profesores u otras actividades no académicas en tiempo lectivo, así como situaciones que dificultan las posibilidades de mejoramiento académico de los docentes de secundaria, entre las que se pueden mencionar:

Falta de permisos para los profesores, para asistir a reuniones académicas o seminarios con especialistas en la materia, por considerarse como pérdida de "tiempo".

- Falta de presupuesto para proyectos relacionados con el mejoramiento de la educación.

Poco conocimiento sobre la importancia del aprendizaje de lenguas extranjeras. 
Es conveniente mencionar que mediante la aplicación del pre-test y del post-test, se detectó que un $18,35 \%$ de los alumnos que conformaron la muestra, abandonaron los estudios en sus instituciones: 703 alumnos hicieron el pre-test y 574 el post-test.

\section{Referencias bibliográficas}

Avendaño, P. et al. Allô Méthodo Litografía Camazu, 1994.

Cascante y Salas. Evaluación del método La Fenêtre en una muestra de estudiantes de $7^{\circ}$ año de Enseñanza Secundaria. I.I. M.E.C., 1995.

Cascante, Lilliam. Rapport sur l'élaboration et les résultats des épreuves de diagnostic et de baccalauréat en français. I.I.M.E.C., 1989.

Díaz, G. y Salas. El diferencial Semántico del Idioma Español, 1970.

Hernández y Matarrita. Elaboración de Escalas para medir la Actitud bacia la Fúsica y el Aprendizaje Fúsico. 1989.
Galisson y Coste. Dictionnaire de Didactique des Langues" Hachette, 1976, p. 341.

I.I.M.E.C. Informe final diagnóstico evaluativo de la enseñanza de las Ciencias Generales, 1985.

Lehman et Alvarez. Une approcbe instrumentale de la lecture en français langue étrangère in Le Français dans le Monde, Paris, 1978.

Moirand, Sophie. Situation d'écrit. Paris, 1979.

Pick y López. Cómo investigar en Ciencias Sociales. Trillas, 1984.

I.I.M.E.C. Informe final sobre el diagnóstico de las necesidades de investigación en la eneñanza de Español, Inglés. Francés, Matemática, Estudios Sociales y Educación Física, 1983.

Porcher, L. et al. La civilisation. Paris, 1986. 